\title{
Photometry and models of selected main belt asteroids. VI. 160 Una, 747 Winchester, and 849 Ara $^{\star}$
}

\author{
A. Marciniak ${ }^{1}$, T. Michałowski ${ }^{1}$, R. Hirsch ${ }^{1}$, M. Polińska ${ }^{1}$, K. Kamiński ${ }^{1}$, T. Kwiatkowski ${ }^{1}$, A. Kryszczyńska ${ }^{1}$, \\ R. Behrend ${ }^{2}$, L. Bernasconi ${ }^{3}$, J. Michałowski ${ }^{4}$, S. Starczewski ${ }^{5}$, M. Fagas ${ }^{1}$, and K. Sobkowiak ${ }^{1}$ \\ 1 Astronomical Observatory, Adam Mickiewicz University, Słoneczna 36, 60-286 Poznań, Poland \\ e-mail: aniab@lab.astro.amu.edu.pl \\ 2 Geneva Observatory, 1290 Sauverny, Switzerland \\ 3 Les Engarouines Observatory, 84570 Mallemort-du-Comtat, France \\ ${ }^{4}$ UNIQA Software Service, Gdańska 132, 90-520 Łódź, Poland \\ 5 N. Copernicus Astronomical Center, Polish Academy of Sciences, Bartycka 18, 00-716 Warsaw, Poland
}

Received 3 October 2008 / Accepted 29 January 2009

\section{ABSTRACT}

\begin{abstract}
We present a set of new photometric observations of three main belt asteroids: 160 Una, 747 Winchester, and 849 Ara. This, combined with the available data, allowed us to construct their physical models. The lightcurve inversion method was used to obtain their spins and shapes. We have resolved problems with the rotation period of $160 \mathrm{Una}$, and found it to be $11.033176 \pm 0.000011 \mathrm{~h}$, almost twice the value given in the literature.
\end{abstract}

Key words. techniques: photometric - minor planets, asteroids

\section{Introduction}

Construction of an increasing number of spin and shape models of asteroids allows for statistical studies that reveal many unknown facts about the Solar System's history, including the forces involved. Precise determinations of the spin periods and axis orientations are important in light of recent direct YORPeffect detections (Kaasalainen et al. 2007; Lowry et al. 2007; Durech et al. 2008) and the apparent proofs of the consequences this effect (Slivan 2002).

Traditional dense lightcurve observations are still an abundant source of asteroids' surface properties, as well as of the overall shapes with their various asymmetries, sharp edges, and other global shape features. Various geometries obtained during a few apparitions of a given body allow a full picture of its properties to be obtained. In particular, observations that cover the wide spans of phase angles carry most of the information on its shape. They allow construction of models based on fewer apparitions than the usual near-opposition lightcurves, which means a shorter data gathering period.

This is the sixth paper in a series that gives three new asteroid models constructed with the lightcurve inversion method. We combined data available in the literature, taken from the Asteroid Photometric Catalogue (Lagerkvist et al. 2001), with our own observations gathered over a campaign of a few years conducted at the Borowiec Observatory, and with amateur observations.

The models presented in this work will be added to two databases. An up-to-date database of all published asteroids spin parameters is available at our website: http: //wwW. astro.amu.edu.pl/Science/Asteroids/

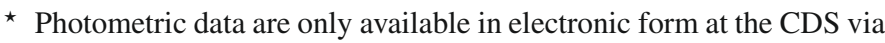
anonymous ftp to cdsarc.u-strasbg.fr $(130.79 .128 .5)$ or via http://cdsweb.u-strasbg.fr/cgi-bin/qcat?J/A+A/498/313
(Kryszczyńska et al. 2007), and the models from lightcurve inversion are collected at: http://astro.troja.mff.cuni. $\mathrm{cz} /$ projects/asteroids3D.

\section{Photometry of three main belt asteroids}

Three main belt asteroids, 160 Una, 747 Winchester, and 849 Ara, were observed photometrically over an eight-year span, for a total of 60 nights. Most of these observations were conducted at the Borowiec Station of the Poznan Astronomical Observatory in Poland. The rest of the lightcurve data come from SAAO in South Africa and from amateur observers from France. It is very beneficial to combine the data from various observatories, even during the same apparition.

The equipment and the description of the reduction procedure can be found in the first paper of the present series: Michałowski et al. (2004). The subsequent papers with different asteroid models are Michałowski et al. (2005, 2006) and Marciniak et al. (2007, 2008). Almost all of the data were collected using small telescopes, like our $40-\mathrm{cm}$ Newtonian one at the Borowiec Station.

In the asteroid observations from mid-northern latitudes, there is always a problem with the lack of data from the Southern Hemisphere. Asteroids are unobservable over long parts of their orbital paths which results in poor longitude coverage. Since the time when we started observations at SAAO in South Africa three years ago, these gaps have been filled successfully (Descamps et al. 2007; Marciniak et al. 2008). Thanks to these observations, we managed to determine unique rotation period of asteroid 160 Una, which turned out to be almost two times longer than previously thought (see Sect. 2.1).

Aspect data for all of the observing runs are presented in Table 1. After the date of observation, given as the mid-time of 
Table 1. Aspect data.

\begin{tabular}{|c|c|c|c|c|c|c|}
\hline Date (UT) & $(\mathrm{AU})$ & (AU) & $\begin{array}{l}\text { Phase } \\
\text { angle } \\
\left({ }^{\circ}\right)\end{array}$ & \multicolumn{2}{|c|}{$\lambda_{(\mathrm{J} 2000)} \beta$} & Obs. \\
\hline \multicolumn{7}{|l|}{160 Una } \\
\hline 20000925.1 & 2.5936 & 1.6284 & 7.60 & 22.25 & 0.60 & Bor \\
\hline 20000927.9 & 2.5922 & 1.6160 & 6.34 & 21.68 & 0.68 & Bor \\
\hline 20000930.1 & 2.5911 & 1.6081 & 5.38 & 21.24 & 0.74 & Bor \\
\hline 20011214.2 & 2.6383 & 2.0184 & 19.08 & 143.31 & 4.52 & Bor \\
\hline 20020203.0 & 2.6722 & 1.6897 & 2.11 & 137.09 & 4.83 & Bor \\
\hline 20020204.0 & 2.6729 & 1.6896 & 1.90 & 136.84 & 4.82 & Bor \\
\hline 20020205.1 & 2.6736 & 1.6898 & 1.78 & 136.60 & 4.81 & Bor \\
\hline 20051005.1 & 2.5443 & 2.0534 & 21.98 & 84.11 & 3.80 & Bor \\
\hline 20051007.1 & 2.5444 & 2.0292 & 21.73 & 84.37 & 3.87 & Bor \\
\hline 20051009.0 & 2.5444 & 2.0066 & 21.47 & 84.59 & 3.93 & Bor \\
\hline 20051019.1 & 2.5451 & 1.8925 & 19.75 & 85.37 & 4.30 & Bor \\
\hline 20070326.1 & 2.8284 & 1.8311 & 0.35 & 185.96 & 0.34 & Bor \\
\hline 20070415.9 & 2.8397 & 1.8936 & 8.25 & 181.58 & -0.09 & Bor \\
\hline 20070425.9 & 2.8449 & 1.9636 & 11.77 & 180.06 & -0.28 & Bor \\
\hline 20080605.1 & 2.8786 & 1.9260 & 8.52 & 279.07 & -5.66 & SAAO \\
\hline 20080607.0 & 2.8780 & 1.9154 & 7.86 & 278.75 & -5.70 & SAAO \\
\hline 20080615.1 & 2.8749 & 1.8789 & 4.93 & 277.20 & -5.82 & SAAO \\
\hline \multicolumn{7}{|l|}{747 Winchester } \\
\hline 20020222.1 & 3.8643 & 3.3340 & 13.36 & 215.98 & 20.21 & Bor \\
\hline 20020309.1 & 3.8849 & 3.1765 & 11.37 & 215.12 & 21.59 & Bor \\
\hline 20020311.1 & 3.8875 & 3.1584 & 11.05 & 214.93 & 21.73 & Bor \\
\hline 20020318.1 & 3.8965 & 3.1012 & 9.88 & 214.09 & 22.29 & Bor \\
\hline 20020319.0 & 3.8978 & 3.0942 & 9.71 & 213.95 & 22.36 & Bor \\
\hline 20020402.0 & 3.9148 & 3.0171 & 7.33 & 211.63 & 23.22 & Bor \\
\hline 20020403.0 & 3.9154 & 3.0152 & 7.26 & 211.54 & 23.25 & Bor \\
\hline 20020423.0 & 3.9380 & 2.9997 & 6.02 & 207.36 & 23.71 & Bor \\
\hline 20030629.0 & 3.7997 & 2.9268 & 8.96 & 246.65 & 19.85 & $\mathrm{EnO}$ \\
\hline 20030630.0 & 3.7980 & 2.9322 & 9.15 & 246.49 & 19.77 & $\mathrm{EnO}$ \\
\hline 20060111.1 & 2.5614 & 1.6726 & 11.68 & 142.48 & 0.26 & Bor \\
\hline 20060215.0 & 2.6886 & 1.7164 & 4.64 & 134.14 & 4.22 & Bor \\
\hline 20060222.9 & 2.7173 & 1.7754 & 7.90 & 132.44 & 4.94 & Bor \\
\hline 20060308.9 & 2.7681 & 1.9201 & 12.84 & 130.26 & 5.97 & Bor \\
\hline 20060322.9 & 2.8184 & 2.1043 & 16.40 & 129.36 & 6.71 & Bor \\
\hline 20070316.1 & 3.7969 & 2.9511 & 8.97 & 205.15 & 21.84 & Bor \\
\hline 20070323.0 & 3.8085 & 2.9184 & 7.71 & 203.93 & 22.30 & Bor \\
\hline 20070327.1 & 3.8151 & 2.9053 & 7.06 & 203.15 & 22.52 & Bor \\
\hline 20070328.1 & 3.8167 & 2.9027 & 6.91 & 202.95 & 22.56 & Bor \\
\hline 20070330.0 & 3.8197 & 2.8987 & 6.65 & 202.58 & 22.65 & Bor \\
\hline 20070413.0 & 3.8415 & 2.8997 & 5.90 & 199.62 & 23.01 & Bor \\
\hline 20070427.0 & 3.8619 & 2.9559 & 7.43 & 196.79 & 22.88 & Bor \\
\hline 20070427.9 & 3.8632 & 2.9615 & 7.58 & 196.62 & 22.86 & Bor \\
\hline 20080424.0 & 3.9630 & 3.1446 & 9.50 & 249.31 & 21.78 & Bor \\
\hline 20080427.0 & 3.9603 & 3.1175 & 8.99 & 248.88 & 21.92 & Bor \\
\hline 20080507.0 & 3.9512 & 3.0439 & 7.31 & 247.25 & 22.26 & Bor \\
\hline 20080513.0 & 3.9455 & 3.0122 & 6.44 & 246.13 & 22.38 & Bor \\
\hline 20080515.0 & 3.9435 & 3.0035 & 6.20 & 245.74 & 22.40 & Bor \\
\hline 20080520.0 & 3.9385 & 2.9873 & 5.77 & 244.74 & 22.42 & Bor \\
\hline 20080530.0 & 3.9279 & 2.9752 & 5.81 & 242.68 & 22.28 & Bor \\
\hline \multicolumn{7}{|l|}{849 Ara } \\
\hline 20040711.0 & 2.5940 & 1.8935 & 19.14 & 341.07 & 27.14 & $\mathrm{EnO}$ \\
\hline 20040713.0 & 2.5958 & 1.8790 & 18.79 & 341.06 & 27.37 & $\mathrm{EnO}$ \\
\hline 20040715.0 & 2.5978 & 1.8643 & 18.41 & 341.03 & 27.61 & $\mathrm{EnO}$ \\
\hline 20060117.8 & 3.5196 & 3.0051 & 14.81 & 51.57 & -7.26 & Bor \\
\hline 20060122.7 & 3.5263 & 3.0810 & 15.30 & 51.72 & -7.34 & Bor \\
\hline 20060127.8 & 3.5331 & 3.1607 & 15.68 & 51.99 & -7.41 & Bor \\
\hline 20060210.9 & 3.5516 & 3.3890 & 16.13 & 53.35 & -7.58 & $\mathrm{EnO}$ \\
\hline 20060211.8 & 3.5528 & 3.4044 & 16.13 & 53.47 & -7.59 & $\mathrm{EnO}$ \\
\hline 20070203.9 & 3.7698 & 2.9236 & 8.73 & 107.14 & -23.33 & Bor \\
\hline 20070307.8 & 3.7645 & 3.2315 & 13.78 & 104.18 & -21.54 & Bor \\
\hline 20070310.8 & 3.7637 & 3.2689 & 14.10 & 104.16 & -21.33 & Bor \\
\hline 20080329.9 & 3.3858 & 2.5052 & 9.27 & 164.57 & -22.51 & Bor \\
\hline 20080408.9 & 3.3693 & 2.5538 & 11.37 & 162.98 & -21.56 & Bor \\
\hline 20080423.8 & 3.3441 & 2.6652 & 14.28 & 161.55 & -19.88 & Bor \\
\hline
\end{tabular}

Observatory Code: Bor - Borowiec; EnO - Les Engarouines Observatory; SAAO - South African Astronomical Observatory.

the lightcurve, there follow the distances from the object to the Sun and to the Earth in AU. In the fourth column there is the Sunasteroid-Earth phase angle, and the other two columns show the
Table 2. Asteroid parameters.

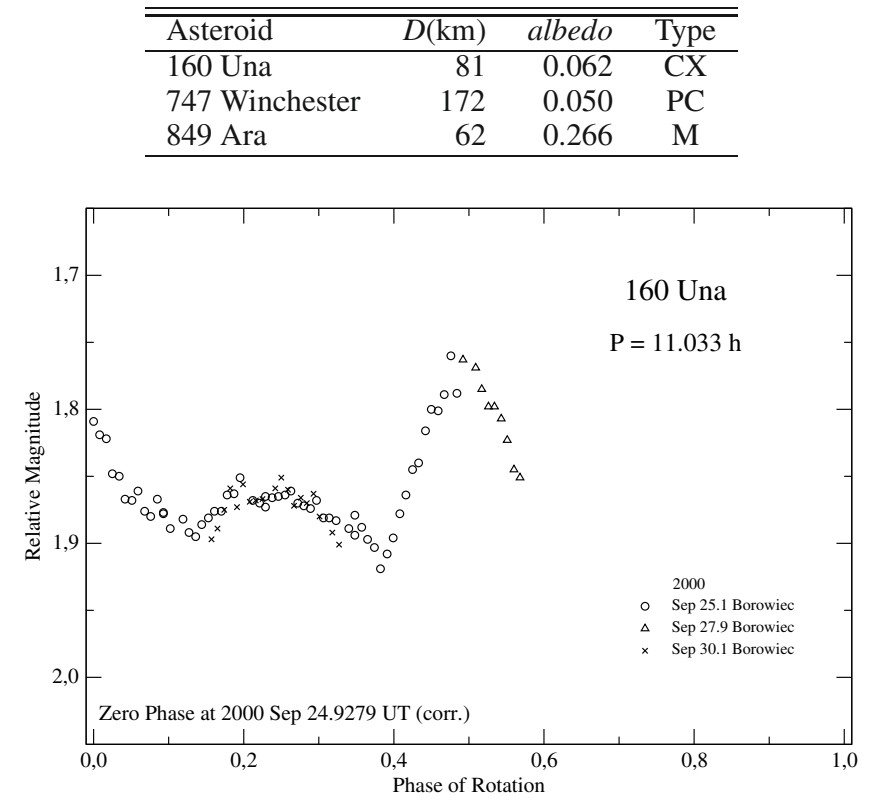

Fig. 1. Composite lightcurve of 160 Una in 2000.

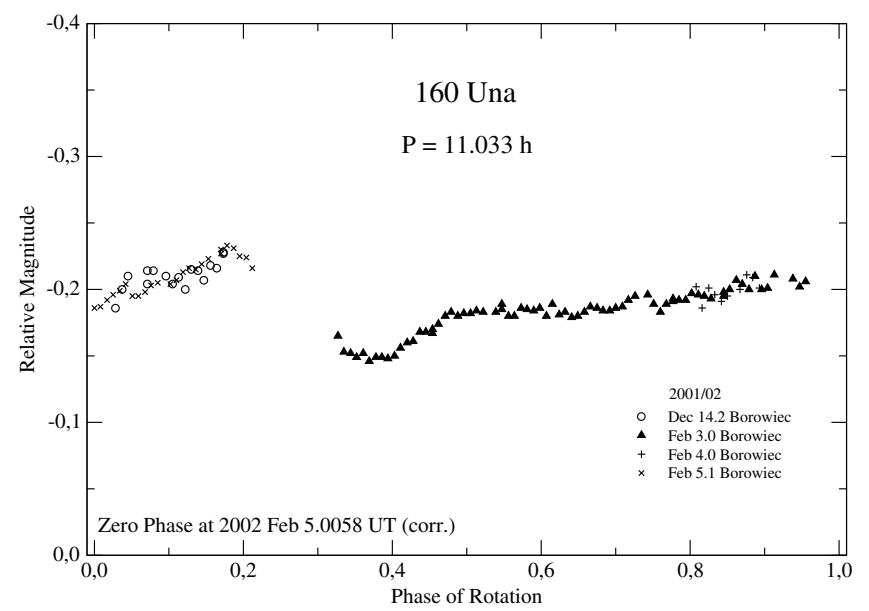

Fig. 2. Composite lightcurve of 160 Una in 2001/2002.

$J 2000$ ecliptic coordinates of a given object at any given moment. The last column contains the observatory code.

Table 2 shows the physical properties of three asteroids. After the object's name there is its IRAS diameter, albedo, and the taxonomic type. The data for this table come from The Small Bodies Node of the NASA Planetary Data System (http:// pdssbn.astro.umd.edu/), where the diameters and albedos we cite come from the IRAS Minor Planet Survey (Tedesco et al. 2004), and the taxonomic classifications are given after Tholen (1989).

Our observations are presented in the form of composite lightcurves, one for each apparition (Figs. 1-14). The composites were created by means of vertical shift, to obtain the smallest possible scatter, with a fixed synodic period, that is shown in the graphs. Different symbols denote different observing runs. The vertical scale, which is the same for all the lightcurves of one object, is the relative magnitude, while the horizontal scale is the phase of rotation. Such compositing allows one to find the first approximation of the rotation period for the inversion procedure. 


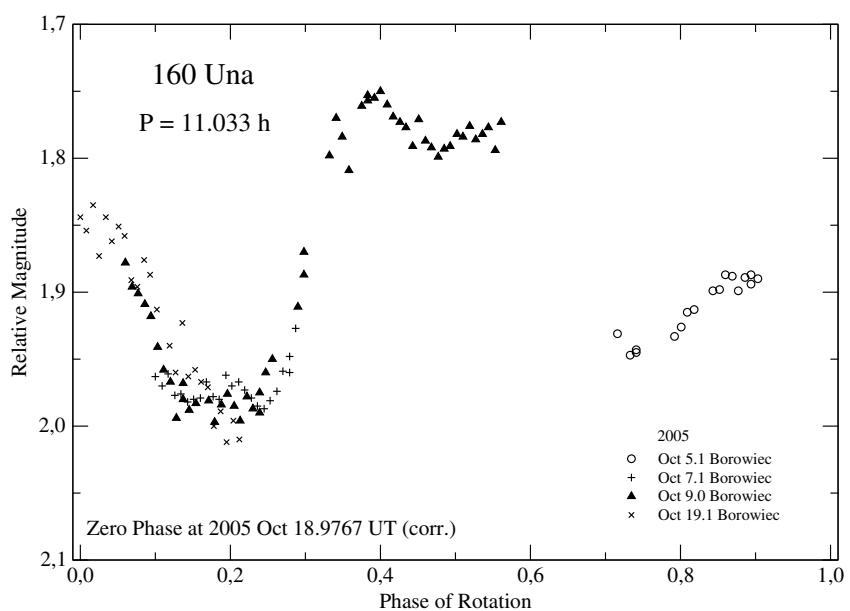

Fig. 3. Composite lightcurve of 160 Una in 2005.

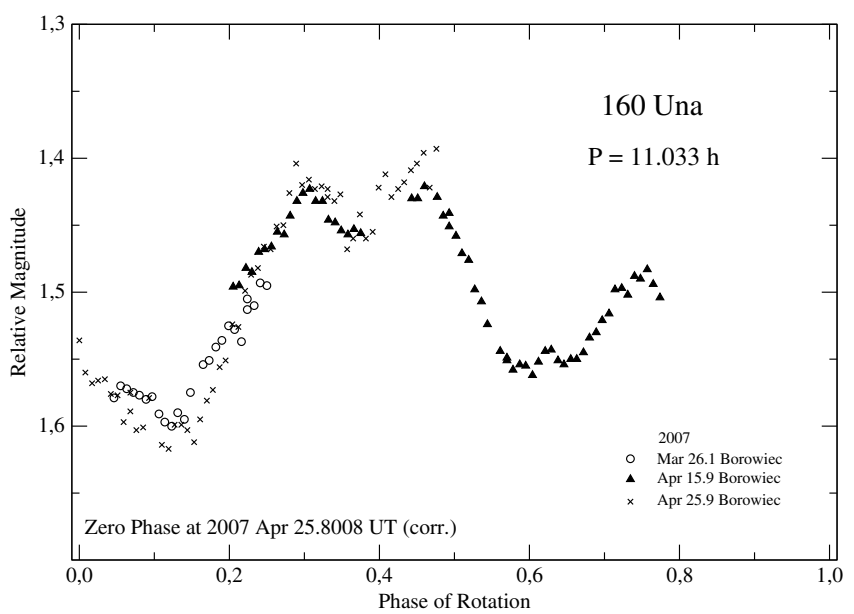

Fig. 4. Composite lightcurve of 160 Una in 2007.

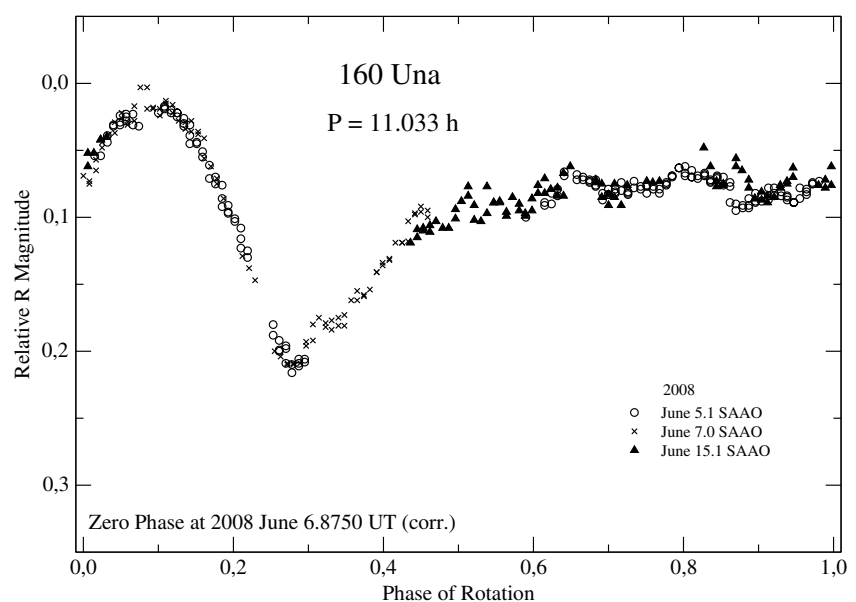

Fig. 5. Composite lightcurve of 160 Una in 2008.

\subsection{Una}

Una was first observed photometrically by Harris et al. (1999). On three consecutive nights in October 1982, the authors obtained three sparse lightcurves, later composited with the $5.55 \pm$ $0.02 \mathrm{~h}$ period, which they considered the most probable, although they did not rule out periods that were two or three times longer. The 0.10 mag amplitude lightcurve was asymmetric, with two "maxima" very close to each other and with shallow

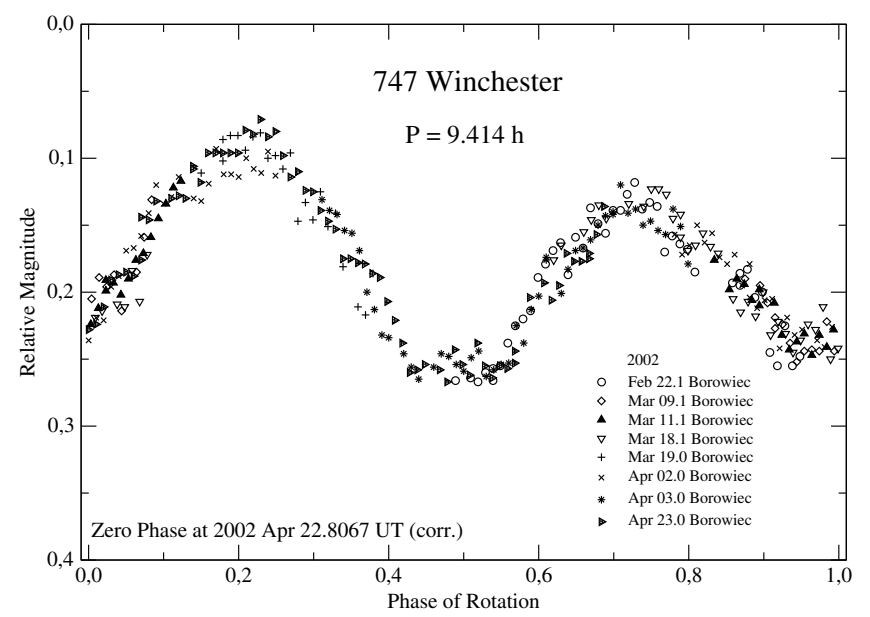

Fig. 6. Composite lightcurve of 747 Winchester in 2002.

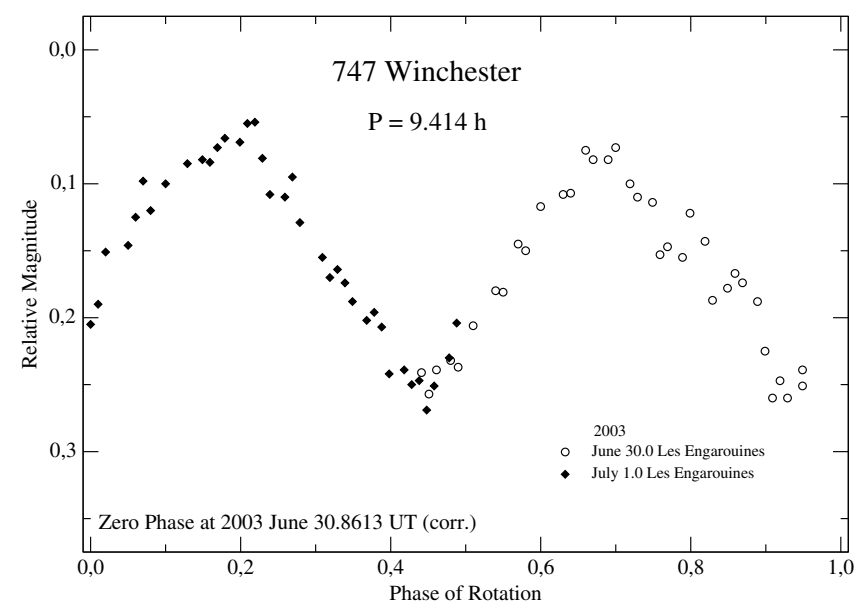

Fig. 7. Composite lightcurve of 747 Winchester in 2003.

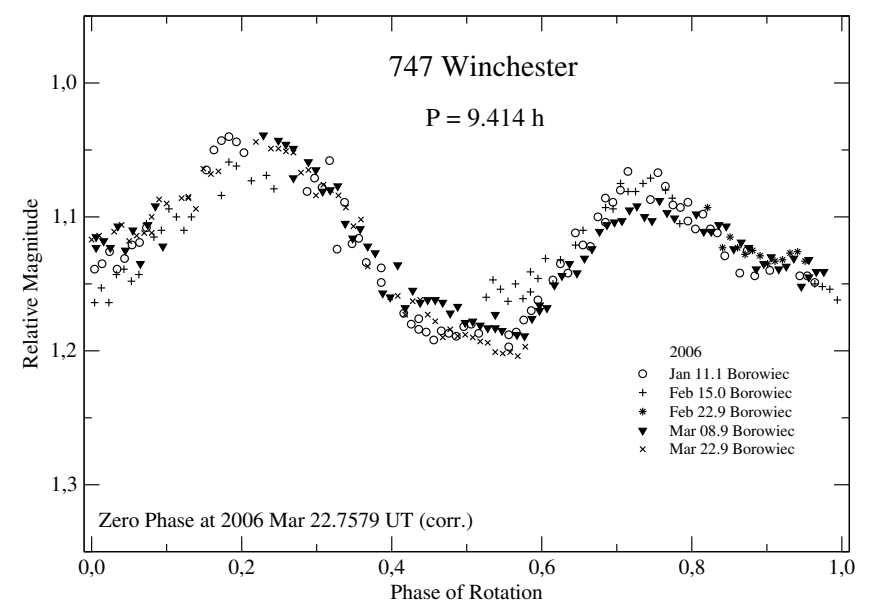

Fig. 8. Composite lightcurve of 747 Winchester in 2006.

minimum between them. The authors also gave its $H-G$ values as $H=9.050 \pm 0.004$ and $G=0.05 \pm 0.04$. Next observations of this object were made by DiMartino et al. (1994) on two consecutive nights in October 1991, almost at the same place on this asteroid's orbit, due to its 9-year commensurability with the Earth's year. Their clumpy lightcurves were composited with a similar $5.61 \pm 0.01 \mathrm{~h}$ period, had an amplitude of $0.14 \pm 0.01 \mathrm{mag}$, and looked more symmetric than the previous 


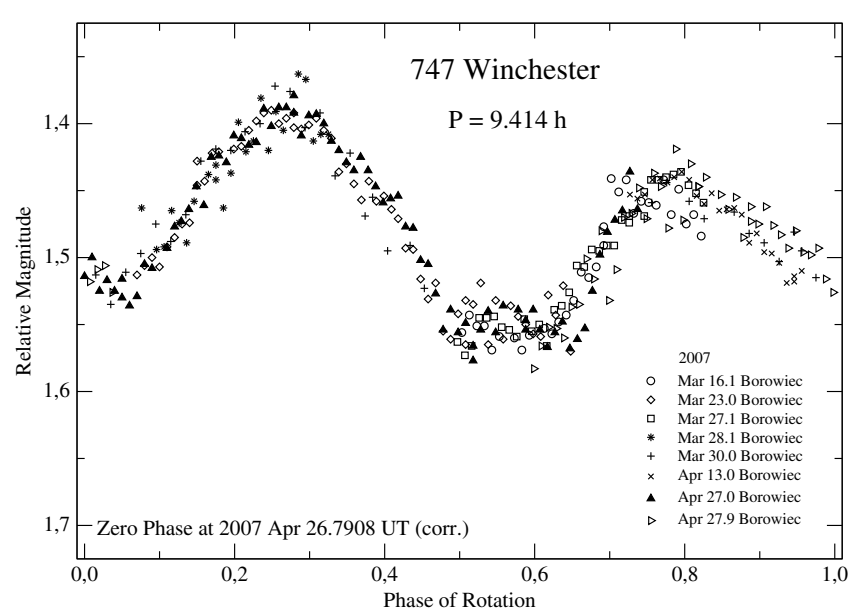

Fig. 9. Composite lightcurve of 747 Winchester in 2007.

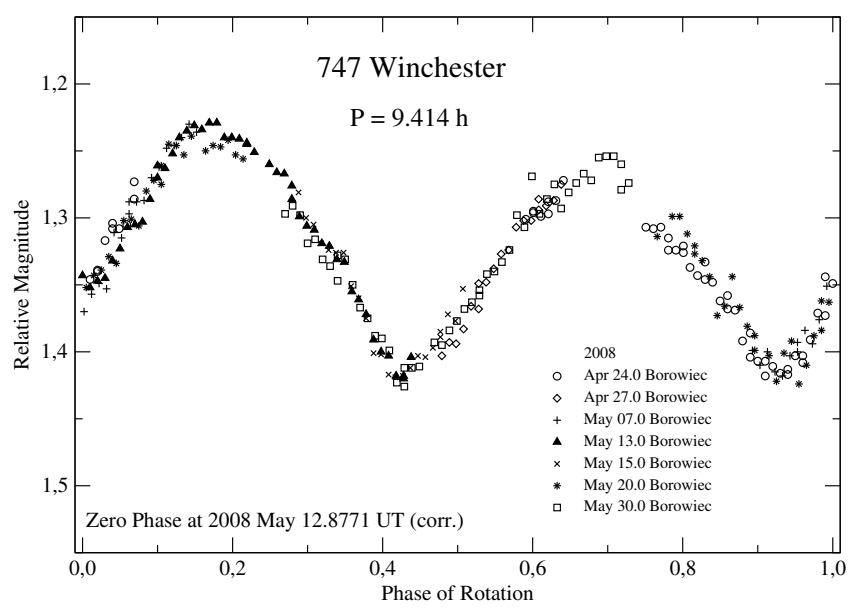

Fig. 10. Composite lightcurve of 747 Winchester in 2008.

one. Finally, Warner (2007b) estimated Una's $H-G$ parameters as $H=8.95 \pm 0.02$ and $G=0.05 \pm 0.05$.

We observed Una at five more apparitions: 2000, 2001/2002, 2005,2007 , and 2008. We usually restricted our observing runs according to its period from the literature: $5.61 \mathrm{~h}$. But after the 2002 apparition, it was clear that the period must be longer than $6 \mathrm{~h}$. Finally, we found that only a much longer period of $11.033 \pm 0.001 \mathrm{~h}$ fits all the apparitions, so that is the period for all the composite lightcurves shown in Figs. 1 to 5. In most of the composites, the individual parts of the lightcurve were too short or not overlapping, so they were arbitrarily shifted in the vertical axis. Asteroid Una showed unusual, flat lightcurves with one pair of extrema in some apparitions, and bimodal curves with wide extrema in the other. The amplitudes ranged from 0.08 to $0.25 \mathrm{mag}$.

\subsection{Winchester}

The first observations of Winchester come from the years 1970 and 1973. Vesely \& Taylor (1985) gave those two lightcurves, the first obtained on one night in March 1970 and the other in August 1973. Both of them are rather noisy and partial, with an amplitude not exceeding $0.13 \mathrm{mag}$. On two close nights in July and August 1978 Harris \& Young (1980) obtained two, rather sparse lightcurves of this asteroid, with an amplitude of 0.13 mag. The lightcurves did not cover the whole rotational cycle, so the period of rotation was suggested to be of over

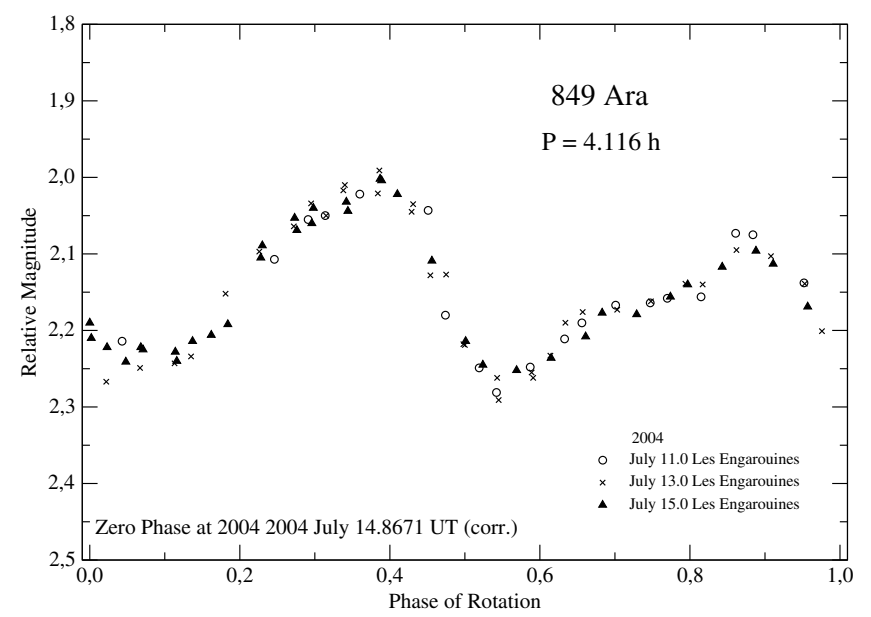

Fig. 11. Composite lightcurve of 849 Ara in 2004.

8 h. Next, Winchester was observed by Zappala et al. (1983). On six nights in February 1980 this object produced an asymmetric, $0.13 \mathrm{mag}$ amplitude lightcurve. The extrema were at slightly different heights. Unfortunately, the data came as a composite lightcurve with a period of $9.40 \pm 0.01 \mathrm{~h}$, which was later corrected, so only one lightcurve of this set fits our model closely. On five nights in March-April 2007, Warner (2007a) observed Winchester obtaining a smooth, asymmetric composite lighcurve with $0.16 \pm 0.02 \mathrm{mag}$ amplitude and $9.4146 \pm 0.0002 \mathrm{~h}$ period. All these data were included in our modelling.

We added five apparitions to the existing set, from the years: 2002, 2003, 2006, 2007, and 2008. All the lightcurves from the separate apparitions (Figs. 6-10) were composited with a synodic period of $9.414 \pm 0.001 \mathrm{~h}$. Winchester always showed regular bi-modal lightcurves with similar amplitudes, ranging from 0.16 to 0.21 mag.

\subsection{Ara}

The only available lightcurves of this object can be found in Harris et al. (1992). There is one dense and four sparse lightcurves, coming from May-June 1981. The period determined by the authors was $4.11643 \pm 0.00005 \mathrm{~h}$, this precision followed from its shortness and a month-long observing span. The lightcurve itself had 0.34 mag amplitude and one of the maxima was asymmetrically shaped. The authors provided also the $H-G$ values as $8.33 \pm 0.01$ and $0.21 \pm 0.01$, respectively.

Our observations of this asteroid spanned four apparitions: 2004, 2006, 2007, and 2008. The period $4.116 \pm 0.001 \mathrm{~h}$ has been confirmed and it fits all the composite lightcurves shown in Figs. 11-14. The look of the lightcurves changed profoundly from one apparition to the next, from a slightly irregular bimodal lightcurve to the ones where one of the minima is almost flat. The amplitudes were in the range of 0.20 to $0.57 \mathrm{mag}$.

\section{Pole and shape results}

To construct the spin and shape models, we used the lightcurve inversion method, whose details can be found in Kaasalainen \& Torppa (2001) and Kaasalainen et al. (2001, 2002a). The procedure uses all the available brightness measurements directly. In the iteration process, the shape model and its spin axis are changed so as to reproduce the observed lightcurves as closely as possible. The resulting fits are usually very good, as the model can even reproduce the small features of the lightcurves. The 


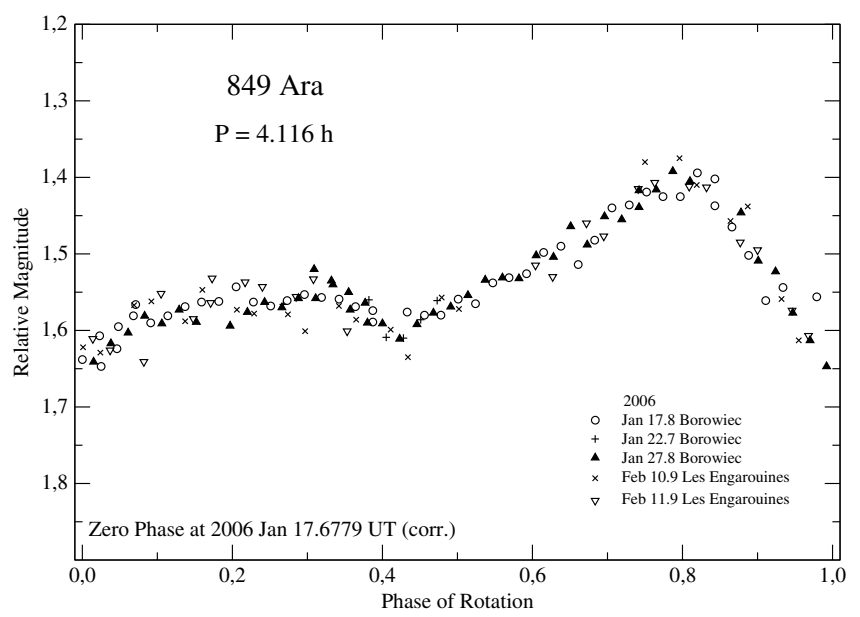

Fig. 12. Composite lightcurve of 849 Ara in 2006.

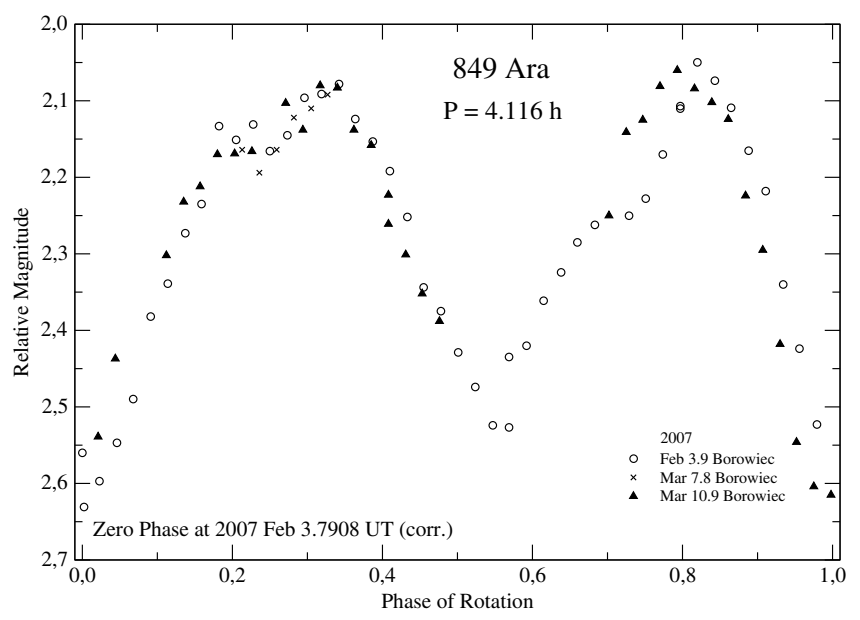

Fig. 13. Composite lightcurve of 849 Ara in 2007.

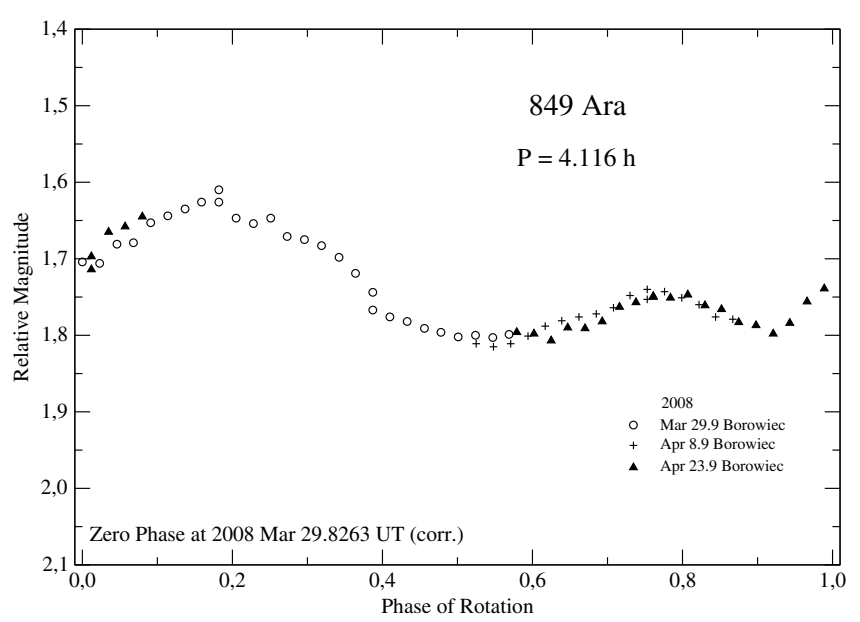

Fig. 14. Composite lightcurve of 849 Ara in 2008.

key requirement for the data is the possibly widest span of the observing geometries. This may not always be the case, as the example of 747 Winchester shows (see below).

Table 3 presents the resulting model parameters. In the first column there is the asteroid's sidereal period of rotation, with an uncertainty restricted to the last digit. Then both pole solutions follow with their J2000 ecliptic longitude $\lambda_{\mathrm{p}}$ and latitude $\beta_{\mathrm{p}}$. The negative value of $\beta_{\mathrm{p}}$ indicates a retrograde rotation, which was confirmed by calculating obliquities $\beta_{\mathrm{p}}$ with respect to the orbits of individual objects. The next three columns of Table 3 give the observing span in years, the number of apparitions, and the number of lightcurves that were used to construct the model. The last two columns show the method used to construct the model and the reference ("L (com)" denotes the lightcurve inversion applied to a combined dataset, with sparse datapoints and a few dense lightcurves). For 747 Winchester and 849 Ara, the pole results have been previously reported and are listed in Table 3 before the present solution.

We give the error values next to the pole coordinates, as they turned out to be unusual. Usually, the uncertainty of the pole solution in the lightcurve inversion is around $\pm 5^{\circ}$ on the celestial sphere. In this method, the solution errors can only be estimated by starting the procedure with various parameters and determining the scatter of the proper solutions. (In Torppa et al. 2003 and Kaasalainen \& Durech 2007 one can find more details on errors in the lightcurve inversion.) All three cases are described below with their errors.

To demonstrate how the model lightcurves fit the observed ones, we present lightcurves coming from three apparitions superimposed on the model lightcurves covering the whole rotational phase (Figs. 15, 17 and 19). The angles $\phi, \phi_{0}$, and $\alpha$ denote the aspect angles of the Earth (as the angle between asteroid's north pole and the direction to the Earth), the Sun, and the phase angle at the epoch of observation, respectively.

\subsection{Una}

The model of 160 Una was successfully constructed with the newly found period of $11.033165 \mathrm{~h}$. Twenty-four lightcurves taken over 7 apparitions were used (from 1982, 1991, 2000, 2001/2002, 2005, 2007, and 2008). Three of the apparitions happened at almost the same place on its orbit, so the actual number of different apparitions was five. This asteroid shows interesting changes in lightcurves, switching from a bimodal look with ditches in maxima to flat lightcurves with a wavy pair of extrema in them. The shape model that reproduces such data has a triangular polar cross-section (Fig. 16) and rather low pole inclination (Table 3), which allows large variations in the visible parts of the surface with the changing aspect. The interesting feature of this asteroid is that its amplitudes sometimes get smaller in aspects close to equatorial, while growing bigger in aspect closer to polar ones (Fig. 15). We usually expect the opposite. For most asteroids the amplitudes of the lightcurves increase at equatorial aspects. But this object's shape is so far from ellipsoidal that we observe smaller amplitudes and the disappearance of one pair of extrema in the equatorial aspects, not close to polar ones. Una proved to be a retrograde rotator. This is the first complete model of this asteroid.

\subsection{Winchester}

The 4-cycle semi-commensurability with the Earth's year makes 747 Winchester a difficult case. It was not until we had 44 lightcurves coming from 9 apparitions of this object (1970, $1973,1978,1980,2002,2003,2006,2007$, and 2008) that we managed to obtain its unique model. Although the rotation period is precisely determined, the pole solution (Table 3 ) and the shape model's vertical dimension (Fig. 18) are not fully constrained. The error in the longitude of the pole solutions reaches $15^{\circ}$, while it is $10^{\circ}$ in latitude, probably due to the restricted viewing geometry of this asteroid. New observations are not 
Table 3. Spin models.

\begin{tabular}{|c|c|c|c|c|c|c|c|c|c|}
\hline \multirow{2}{*}{$\begin{array}{r}\text { Sidereal } \\
\text { period (hours) }\end{array}$} & \multicolumn{2}{|c|}{ Pole 1} & \multicolumn{2}{|c|}{ Pole 2} & \multirow{2}{*}{$\begin{array}{c}\text { Observing span } \\
\text { (years) }\end{array}$} & \multirow[t]{2}{*}{$N_{\text {app }}$} & \multirow[t]{2}{*}{$N_{\mathrm{lc}}$} & \multirow[t]{2}{*}{ Method } & \multirow[t]{2}{*}{ Reference } \\
\hline & $\lambda_{\mathrm{p}}$ & $\beta_{\mathrm{p}}$ & $\lambda_{\mathrm{p}}$ & $\beta_{\mathrm{p}}$ & & & & & \\
\hline 160 Una & & & & & & & & & \\
\hline 11.033176 & $125^{\circ}$ & $-33^{\circ}$ & $308^{\circ}$ & $-41^{\circ}$ & 1982-2008 & 7 & 24 & $\mathrm{~L}$ & Present work \\
\hline \pm 0.000011 & $\pm 5^{\circ}$ & $\pm 2^{\circ}$ & $\pm 5^{\circ}$ & $\pm 2^{\circ}$ & & & & & \\
\hline \multicolumn{10}{|l|}{747 Winchester } \\
\hline \multirow[t]{2}{*}{9.402} & $27^{\circ}$ & $+50^{\circ}$ & & & 1970-1980 & 4 & 5 & EAM & Michałowski (1993) \\
\hline & & & $353^{\circ}$ & $39^{\circ}$ & 1970-1980 & 4 & 5 & EA & De Angelis (1995) \\
\hline 9.414807 & $166^{\circ}$ & $-44^{\circ}$ & $296^{\circ}$ & $-61^{\circ}$ & 1970-2008 & 9 & 44 & $\mathrm{~L}$ & Present work \\
\hline \pm 0.000005 & $\pm 15^{\circ}$ & $\pm 10^{\circ}$ & $\pm 15^{\circ}$ & $\pm 10^{\circ}$ & & & & & \\
\hline \multicolumn{10}{|l|}{849 Ara } \\
\hline 4.116391 & $17^{\circ}$ & $-10^{\circ}$ & $213^{\circ}$ & $-33^{\circ}$ & 1981-2003 & $1^{*}$ & 5 & $\mathrm{~L}(\mathrm{com})$ & Ďurech et al. (2009) \\
\hline 4.116391 & $10^{\circ}$ & $-25^{\circ}$ & $223^{\circ}$ & $-40^{\circ}$ & 1981-2008 & 5 & 20 & $\mathrm{~L}$ & Present work \\
\hline \pm 0.000001 & $\pm 1^{\circ}$ & $\pm 1^{\circ}$ & $\pm 3^{\circ}$ & $\pm 3^{\circ}$ & & & & & \\
\hline
\end{tabular}

* 1 appartition with 5 dense lightcurves +133 sparse data points.
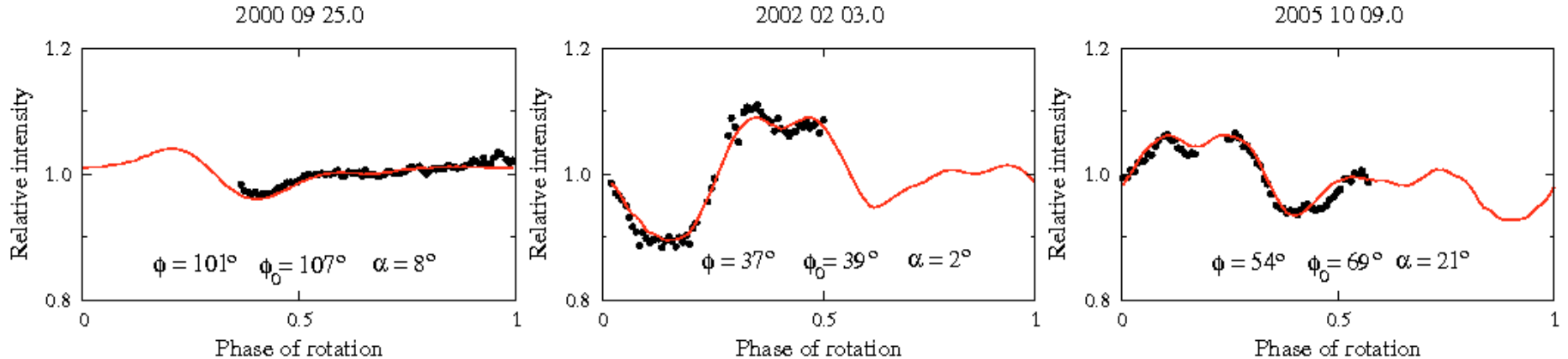

Fig. 15. Observed lightcurves (points) superimposed on the lightcurves created by a model (curves) at the same epochs for 160 Una.

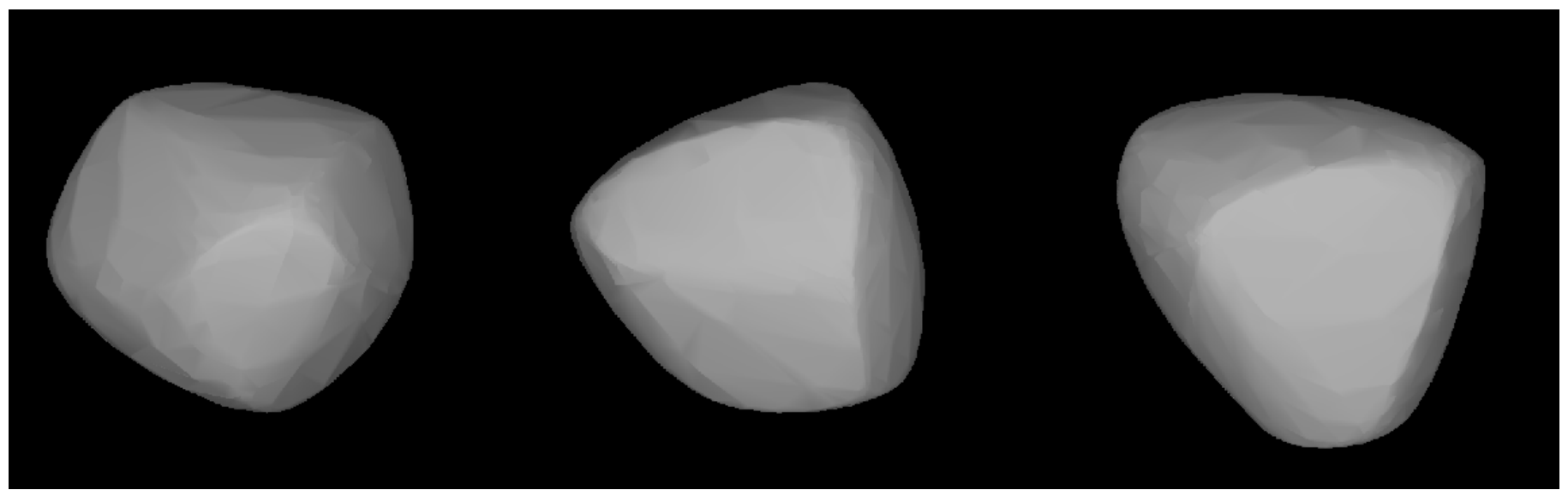

Fig. 16. Shape model of 160 Una, shown at equatorial viewing and illumination geometry, with rotational phases $90^{\circ}$ apart (two pictures on the left) and the pole-on view on the right.

20020423.0

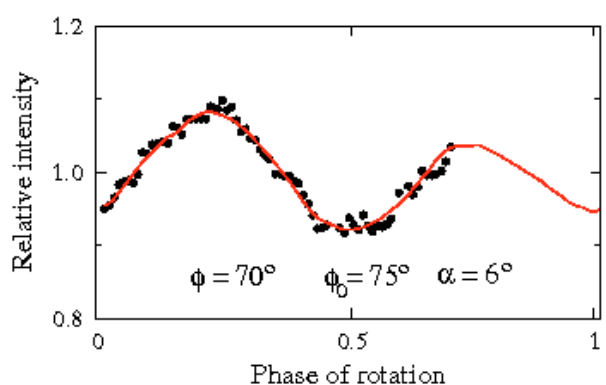

20060111.1

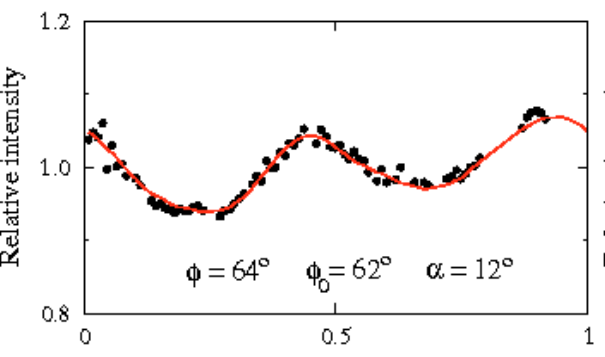

Phase of rotation
20080530.0

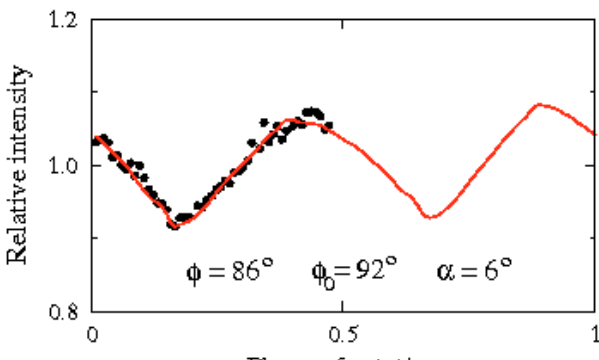

Phase of rotation

Fig. 17. Observed versus modelled lightcurves for 747 Winchester. 


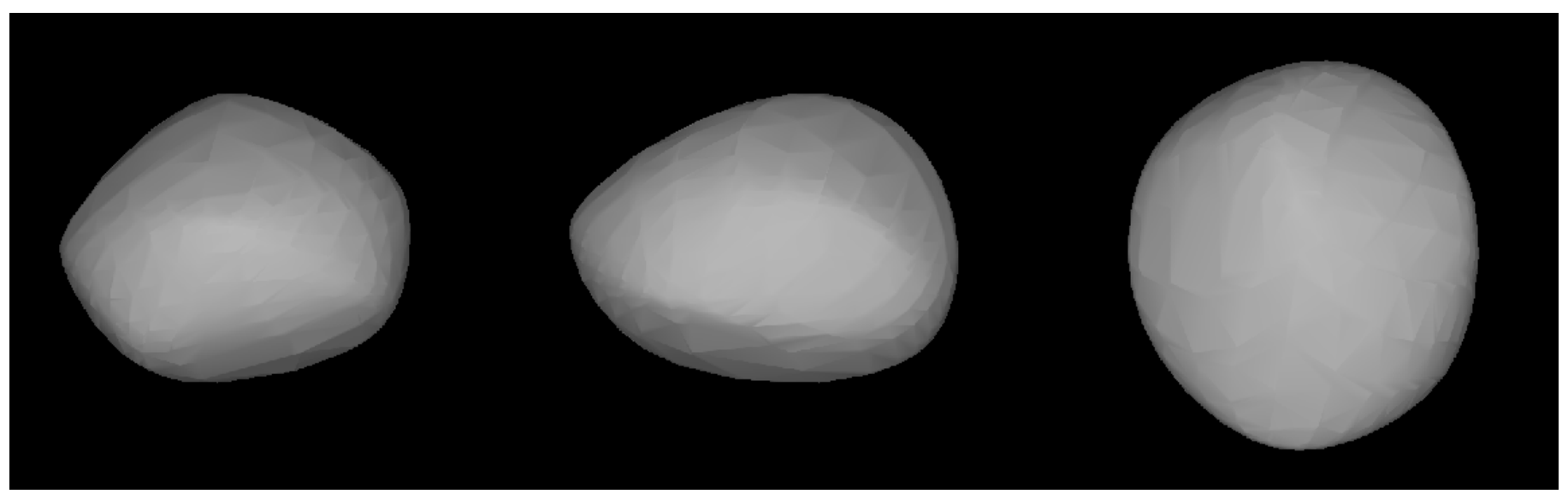

Fig. 18. Shape model of 747 Winchester.

20040713.0

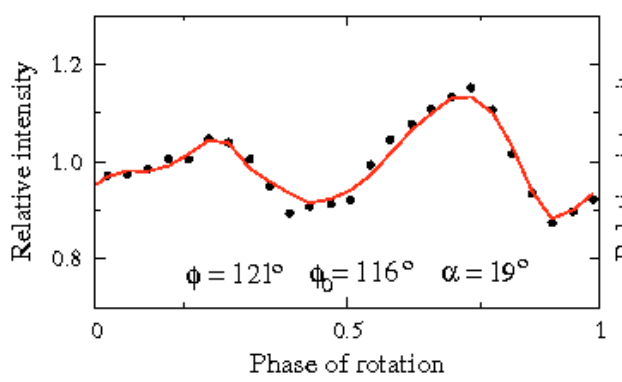

Fig. 19. Observed versus modelled lightcurves for 849 Ara.

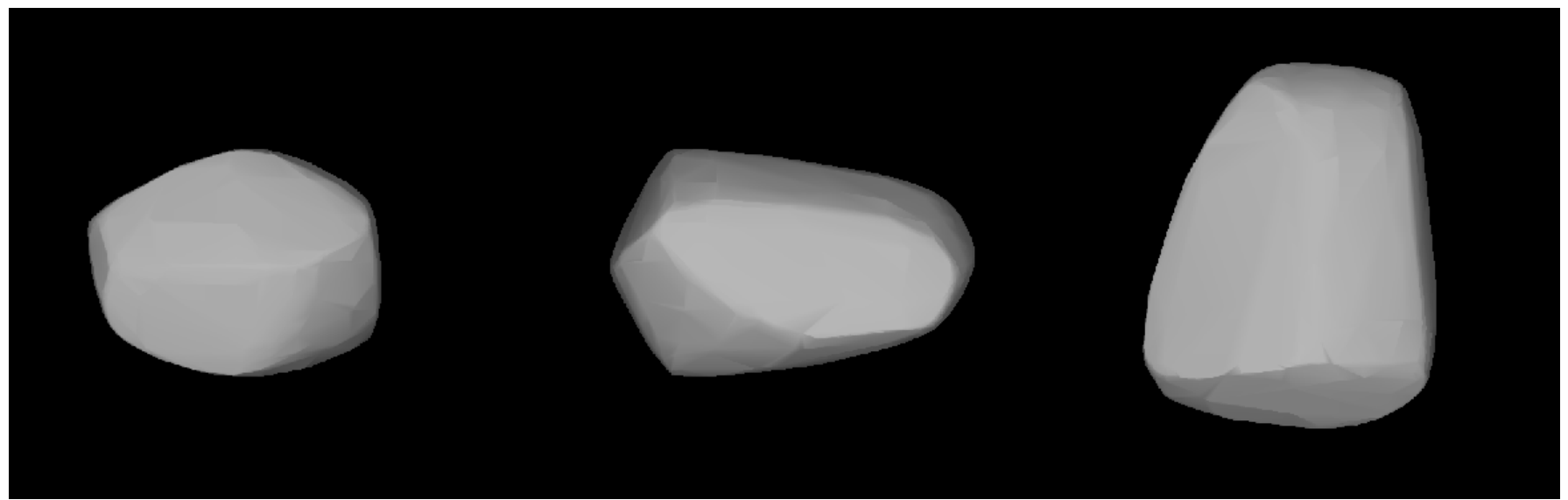

Fig. 20. Shape model of 849 Ara

expected to improve the model until about ten years from now, when the repeating pattern shifts considerably along the orbit.

Our pole solution differs a lot from the previous pole determinations made by Michałowski (1993) or by De Angelis (1995). However, the latter author wrote that the sense of rotation has not been determined, but he did not give an alternative pole solution (around $\lambda_{\mathrm{p}}=173^{\circ}, \beta_{\mathrm{p}}=-39^{\circ}$ ), which would then be close to our solution, because Winchester seems to be a retrograde rotator.

\subsection{Ara}

For a change 894 Ara was a graceful object to model and to observe. With its lightcurves of unusual shape and evenly distributed apparitions, a unique model already appeared after 5 of them (from 1981, 2004, 2006, 2007, and 2008). Here, the scatter of the pole solutions was unprecedently small, as low as $1^{\circ}$
20060117.8

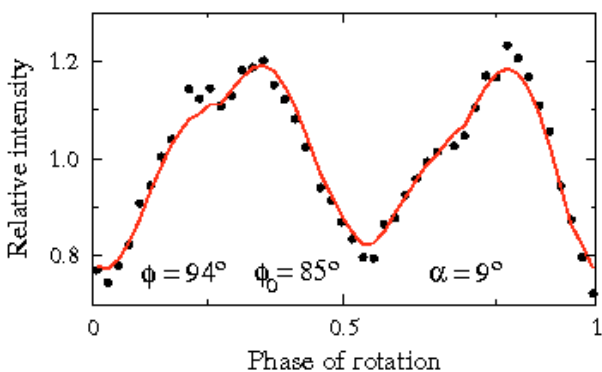

Phase of rotation

20070203.9

Phase of rotation

(n)


by Mikko Kaasalainen and modified by Josef Durech, and is available at http://astro.troja.mff.cuni.cz/projects/asteroids3D.

\section{References}

De Angelis, G. 1995, Planet. Space Sci. 43, 649

Descamps, P., Marchis, F., Michałowski, T., et al. 2007, Icarus, 187, 482

Di Martino, M., Blanco, C., Riccioli, D., \& De Sanctis, G. 1994, Icarus, 107, 269

Durech, J., Vokrouhlicky, D., Kaasalainen, M., et al. 2008, A\&A, 489, L25

Durech, J., Kaasalainen, M., Warner, B., et al. 2009, A\&A, 493, 291

Harris, A. W., \& Young, J. W. 1980, Icarus, 43, 20

Harris, A. W., Young, J. W., Dockweiler, T., et al. 1992, Icarus, 95, 115

Harris, A. W., Young, J. W., Bowell, E., \& Tholen, D. J. 1999, Icarus, 142, 173

Kaasalainen, M., \& Torppa, J. 2001, Icarus, 153, 24

Kaasalainen, M., \& Durech, J. 2007, in Near Earth Objects, our Celestial Neighbors: Opportunity and Risk, ed. A. Milani, G. B. Valsecchi, \& D. Vokrouhlicky (Cambridge: Cambridge University Press), 151

Kaasalainen, M., Torrpa, J., \& Muinonen, K. 2001, Icarus, 153, 37

Kaasalainen, M., Mottola, S., \& Fulchignoni, M. 2002a, In Asteroids III, ed. W. F. Bottke, A. Cellino, P. Paolicchi, \& R. P. Binzel (Univ. Arizona Press), 139
Kaasalainen, M., Torrpa, J., \& Piironen, J. 2002b, A\&A, 383, L19

Kaasalainen, M., Durech, J., Warner, B. D., et al. 2007, Nature, 446, 420 Kryszczyńska, A., La Spina, A., Paolicchi, P., et al. 2007, Icarus, 192, 223

Lowry, S. C., Fitzsimmons, A., Pravec, P., et al. 2007, Science, 316, 272

Lagerkvist, C.-I., Magnusson, P., \& Erikson, E. 2001, Asteroid Photometric Catalogue, fifth update, Uppsala Astronomical Observatory

Marciniak, A., Michałowski, T., Kaasalainen, M., et al. 2007, A\&A, 473, 633

Marciniak, A., Michałowski, T., Kaasalainen, M., et al. 2008, A\&A, 478, 559

Michałowski, T. 1993, Icarus, 106, 563

Michałowski, T., Kwiatkowski, T., Kaasalainen, M., et al. 2004, A\&A, 416, 353

Michałowski, T., Kaasalainen, M., Marciniak, A., et al. 2005, A\&A, 443, 329

Michałowski, T., Kaasalainen, M., Polińska, M., et al. 2006, A\&A, 459, 663

Slivan, S. M. 2002, Nature, 419, 49

Tedesco, E. F., Noah, P. V., Noah, M., \& Price, S. D. 2004, IRAS Minor Planet Survey. IRAS-A-FPA-3-RDR-IMPS-V6.0. NASA Planetary Data System

Tholen, D. J. 1989, In Asteroids II, ed. R. P. Binzel, T. Gehrels, \& M. S. Matthews (Univ. Arizona Press), 1139

Torppa, J., Kaasalainen, M., Michalowski, T., et al. 2003, Icarus, 164, 346

Vesely, C. D., \& Taylor, R. C. 1985, Icarus, 64, 37

Warner, B. D. 2007a, Minor Planet Bull., 34, 104

Warner, B. D. 2007b, Minor Planet Bull., 34, 113

Zappala, V., Scaltriti, F., Di Martino, M. 1983, Icarus, 56, 325 